\title{
Investigation of Dependence between Time-zero and Time-dependent Variability in High- $\kappa$ NMOS Transistors
}

\author{
Mohammad Khaled Hassan and Kaushik Roy \\ School of Electrical and Computer Engineering, Purdue University, West Lafayette, IN, USA
}

\begin{abstract}
Bias Temperature Instability (BTI) is a major reliability concern in CMOS technology, especially with Highdielectric constant (High-K/HK) metal gate (MG) transistors. In addition, the time-independent process-induced variation has also increased because of the aggressive scaling down of devices. As a result, the faster devices at the lower threshold voltage distribution tail experience higher stress, leading to additional skewness in BTI degradation. Since time-dependent dielectric breakdown (TDDB) and stress-induced leakage current (SILC) in NMOS devices are correlated to BTI, it is necessary to investigate the effect of time-zero variability on all of these effects simultaneously. Accordingly, we propose a simulation framework to model and analyze the impact of time-zero variability (in particular, random dopant fluctuations) on different aging effects. For small area devices $\left(\sim 1000 \mathrm{~nm}^{2}\right)$ in $30 \mathrm{~nm}$ technology, we observe significant effects of Random Dopant Fluctuation (RDF) on BTI-induced variability $\left(\sigma_{\Delta V t h}\right)$. In addition, circuit analysis reveals similar trend in performance degradation. However, both TDDB and SILC show weak dependence on RDF. We conclude that the effect of RDF on $V_{t h}$ degradation cannot be disregarded in scaled technology and needs to be considered in variation-tolerant circuit design.
\end{abstract}

Index Terms - BTI, Variability, RDF, High- $\kappa$ dielectrics, TDDB, SILC.

\section{INTRODUCTION}

$\mathrm{I}$ $\mathrm{N}$ order to aggressively downscale devices and suppress the

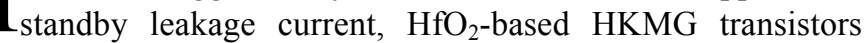
were adopted recently by the IC industry [1]. Their advantages over conventional silicon oxynitride ( $\mathrm{SiON})$ devices have already been demonstrated to a great extent in different literature [1-3]. However, this changeover in the technology roadmap resulted in new failure mechanisms (such as Positive Bias Temperature Instability (PBTI), Stress Induced Leakage Currents (SILC), etc.) that enhanced the complexity of reliability quantification [4-6]. Due to the presence of d-shell electrons and a relatively higher coordination number, high- $\kappa$ oxides are vulnerable to both native and time-dependent, stress-induced defect formation [7-8]. Since $\mathrm{HfO}_{2}$ bulk traps cannot be charged under negative stress voltage, these defects only affect the threshold voltage and leakage current

The authors are with the School of Electrical and Computer Engineering at Purdue University, West Lafayette, IN 47907 (email: khaled@purdue.edu, kaushik@purdue.edu) of NMOSFETs [4]. Along with the time-dependent BTIinduced variations play a critical role in reliability assessment at both the device and circuit levels [9-10]. In sub-45nm technology, the native oxide thickness has decreased to 2 or 3 atomic layers. Consequently, it is important to understand the nature of $\sigma_{\Delta V t h}$ with the change in device dimensions. In addition, the dependence between time-zero and timedependent variability needs to be properly addressed. The devices at the lower process-induced distribution tail performs faster (Fig. 1) due to their low turn-on voltage compared to the nominal threshold voltage, $V_{t h}(0)$. The variation can be up to few sigma values $\left(\sigma_{V t h(0)}\right)$ of the time-zero variation. This increases the stress voltage across the high- $\kappa$ and interfacial layer (IL), causing faster trap generation in the corresponding oxide layers. As an example, in order to comprehend the nature of RDF and temporal BTI evolution and their dependence, it is important to capture the effect of each dopant in the channel region and defect in the bulk oxide layers simultaneously and determine the corresponding effect on $V_{t h}$ degradation. Therefore, a complete modeling framework for RDF as well as BTI variation, considering the fluctuation in number and position of independent dopants and their impact on oxide defects/traps, is necessary for circuit analysis.

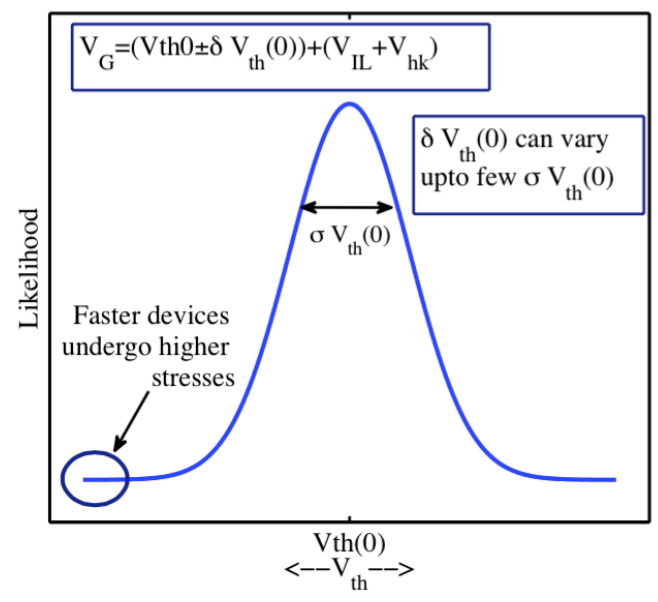

Fig. 1: Time-zero variation $\left(\delta_{V t h(0)}\right)$ with respect to the nominal threshold voltage, $V_{t h}(0)$ can be up to a few $\sigma_{V t h(0)}$. This can cause a non-uniform stress voltage distribution across the oxide layers. 


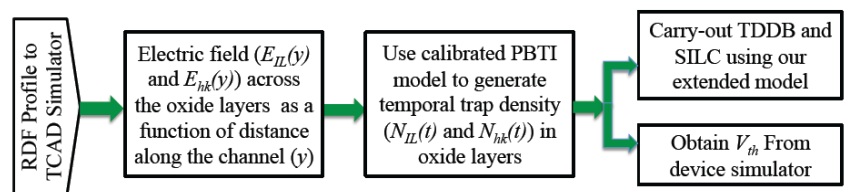

Fig. 2: Our simulation framework.

The rest of the paper is organized as follows. In section II, we explained our modeling and simulation framework. In this section, we have briefly introduced the PBTI model [11] and our extended model in order to analyze time dependent oxide degradation. Section III discuses the possible dependence between RDF and BTI at the device and circuit levels of design. We have also carried out TDDB and SILC analyses of NMOS devices considering this interrelation. Finally, section IV concludes this work.

\section{FRAMEWORK FOR VARIABILITY ANALYSIS}

Fig. 2 shows the framework of our analysis. We have investigated the dependence between time-zero variation (RDF) and time-dependent variability and evaluated its impact on aging effects such as BTI, TDDB, and SILC. Understanding and modeling such an interrelation can be significant in reliability-aware circuit design. In order to properly model the impact of RDF on different aging effects, the non-uniformity of the electric field across the dual oxide layers (HK and IL) along the channel length needs to be considered. To that effect, we have used a TCAD simulator to carry out our device-level simulation [12]. We divided the channel region in $35 \times 30$ number points and extracted the oxide electric field as a function of the channel length. The extracted fields are then incorporated to our BTI trap generation model [11] and calibrated [13]. Subsequently, we integrated our PBTI model to the device simulator in order to analyze the dependence between RDF and PBTI. The TDDB and SILC analyses are carried using our extended trap generation model developed in MATLAB [14].

The NMOS device designed in the TCAD simulator is benchmarked with ITRS 2009 at the 30nm technology node [5]. The thicknesses of the high- $\kappa$ and interfacial layers are $2 \mathrm{~nm}$ and $0.6 \mathrm{~nm}$, respectively. Other design parameters are

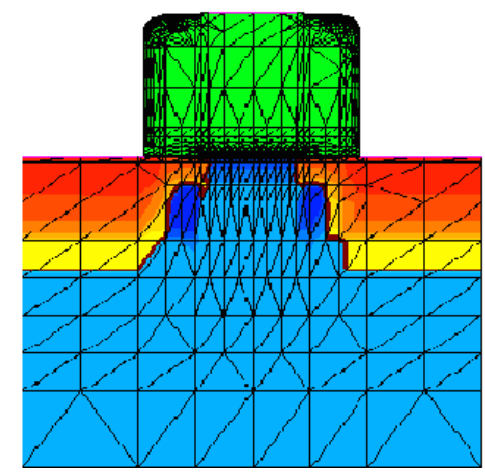

Fig. 3: Cross-section of our simulated NMOS device.
Table 1: NMOS and PMOS design parameters

\begin{tabular}{|l|l|}
\hline Parameter Name & Value \\
\hline Gate Length (L) & $30 \mathrm{~nm}$ \\
\hline Gate Width (W) & $35 \mathrm{~nm}$ \\
\hline Gate Oxide Material & $\mathrm{HfO}_{2}, \mathrm{SiO}_{2}$ \\
\hline Equivalent Oxide Thickness & $0.95 \mathrm{~nm}$ \\
\hline Channel Doping & $2.7 \times 10^{18} / \mathrm{cm}^{3}$ \\
\hline Source/Drain Doping & $6 \times 10^{20} / \mathrm{cm}^{3}$ \\
\hline Halo Doping & $2 \times 10^{19} / \mathrm{cm}^{3}$ \\
\hline Source/Drain Extension & $10 \mathrm{~nm}$ \\
\hline Spacer Length & $20 \mathrm{~nm}$ \\
\hline NMOSFET Gate Workfunction & $4.6 \mathrm{eV}$ \\
\hline PMOSFET Gate Workfunction & $5.06 \mathrm{eV}$ \\
\hline
\end{tabular}

shown in Table 1. The ON and OFF currents of the simulated NMOS device are $1190 \mu \mathrm{A} / \mu \mathrm{m}$ and $100 \mathrm{nA} / \mu \mathrm{m}$, respectively. We have used the drift-diffusion transport model and the unified mobility model from Philips for the simulations [15]. For quantum correction, the device simulator uses the Van Dort model [16]. A detailed explanation of these models and a list of all the parameters used are available in the device user guide of the TCAD simulator [12]. Fig. 3 shows the crosssection of our NMOS device. Unless otherwise specified, the stress voltage and temperature were maintained at $2 \mathrm{~V}$ and $125^{\circ} \mathrm{C}$. We applied a drain voltage of $50 \mathrm{mV}$ in the device simulator and extracted $V_{\text {th }}$ using the maximumtransconductance method, which is a built-in feature of the TCAD simulator [12]. The various parameters used in our reliability analysis are shown in Table 2 . Below we discuss RDF modeling and analysis and different time-dependent aging effects integrated into our simulation framework.

\section{A. Time-zero Variability}

Time-zero fluctuation is comprised of three major components: (1) Random-Dopant Fluctuation (RDF), (2) Mean-Gate Length Deviation (GLD), and (3) Line-Edge Roughness (LER) [18]. Among these three, RDF is considered to have the most adverse effects [18-20]. With the aggressive

Table 2: Parameters used in our simulation

\begin{tabular}{|c|c|c|}
\hline Parameters & Value & Reference \\
\hline$\alpha\left(\mathrm{HfO}_{2}\right)$ & 0.17 & {$[4,13]$} \\
\hline$\alpha\left(\mathrm{SiO}_{2}\right)$ & 0.38 & {$[39]$} \\
\hline$E_{r}\left(\mathrm{HfO}_{2}\right)$ & $0.8 \mathrm{eV}$ & {$[40]$} \\
\hline$E_{r}\left(\mathrm{SiO}_{2}\right)$ & $0.1 \mathrm{eV}$ & {$[41]$} \\
\hline$a_{0}\left(\mathrm{HfO}_{2}\right)$ & $1 \mathrm{~nm}$ & {$[17]$} \\
\hline$a_{0}\left(\mathrm{SiO}_{2}\right)$ & $0.6 \mathrm{~nm}$ & {$[17]$} \\
\hline$\Phi_{B}$ & $3.2 \mathrm{eV}$ & {$[31]$} \\
\hline$\sigma_{n}$ & $10^{-14}-10^{-15} \mathrm{~cm}^{2}$ & {$[27]$} \\
\hline \multicolumn{3}{|c|}{$a_{0}=$ trap size } \\
$\sigma_{n}:$ Trap Cross section \\
$E_{r}:$ Trap Relaxation Energy \\
$\Phi_{B}:$ Barrier height of the electrons \\
\hline
\end{tabular}


scaling down of MOSFETs, the intrinsic variation of $\sigma_{V t h(0)}$ due to the smaller number of discrete implanted dopants and their corresponding random position in the channel becomes more distinct [20-21]. RDF causes a large variation among similar transistors and affects performance at the device level as well as the circuit level of design abstraction [22]. In this work, we have generated RDF profiles considering randomness in the number of dopants using a pseudo-random number generator [14].

$$
N_{R D F}^{i}(0)=\operatorname{Poiss}\left(N_{R D F_{\text {avg }}}(0)\right)_{1 x S} \text {, where } i=1,2, \ldots ., S
$$

where, $N_{R D F}^{i}$ is a randomly drawn number from an $1 \mathrm{xS}$ vector generated by Poisson's distribution. $N_{R D F a v g}$ is the average doping density in the channel region and $S$ is the number of microscopically-similar sample devices. In addition to the number of random dopants, randomness of the dopant's location also plays a significant role on $V_{t h}$ variation. Dopants that are closer to the $\mathrm{Si} / \mathrm{SiO}_{2}$ interface have larger influence in $V_{\text {th }}$ fluctuation [21]. Moreover, Drain-Induced Barrier Lowering (DIBL) increases the standard deviation of $V_{t h}$ in nanoscale transistors that is attributed to RDF. This is because the drain potential can nullify the effect of dopants that are located close to the drain contact; a large number of dopants close to the drain contact can cause high potential profile [22]. The RDF profiles are fed to the TCAD simulator and the locations of the dopants were randomized using the built-in feature of the TCAD simulator.

\section{B. Positive Bias Temperature Instability (PBTI)}

PBTI emerged as a significant reliability concern for High- $\kappa$ NMOS transistors. We proposed a physics-based statistical PBTI model in which the location of each of the traps was considered separately [11]. The trap generation process in dielectric layers depends on stress time $(\mathrm{t})$, temperature $(\mathrm{T})$, and, the applied gate bias $\left(\mathrm{V}_{\mathrm{G}}\right)$ and the number of generated traps $\left(N_{G}\right)$ can be modeled as follows:

$$
N_{G}\left(V_{G}, T, t\right)=A \times\left(V_{G}\right)^{\gamma} \exp \left(-E_{a} / K_{B} T\right) t^{n}
$$

where, $V_{G}$ is the voltage drop across the dielectric layer, $\gamma$ is the voltage acceleration factor, $n$ is the PBTI power exponent, $K_{B}$ is the Boltzmann constant, and $A$ is a proportionality constant and the calibration parameter that depends on the device structure. Typical values of $\mathrm{n}$ and $\gamma$ for PBTI degradation are $\sim 0.17$ and $\sim 5$, respectively [4]. We assumed a random distribution of these traps across the dielectric layers and assigned a stochastic process $S(t)$ to each of the oxide bonds:

$$
S(t)=\left\{\begin{array}{l}
1: \text { if the bond is broken } \\
0: \text { if the bond is tied }
\end{array}\right\}
$$

Therefore, the temporal variation of randomly distributed generated traps can be given as

$$
N_{(G i)_{r}}(t)=\int_{x=0}^{W} \int_{y=0}^{L} \int_{z=0}^{T_{d}} S(t) d x d y d z
$$

where, $W, L$, and $T_{d}$ are respectively the width, length, and, the thickness of the oxide layer. For our simulation, we have discretized the oxide layers along their width, length, and height depending on the defect sizes in the corresponding oxide layers (Table 2).

Using probability theory, we have determined the number of charged traps in the oxide layers. Trapping of carriers is dependent on the generation of traps and therefore is a conditional event. The probability that both trapping and trap generation events can occur at the same location $(x, y, z)$ at a specified time is given by [23]:

$$
P(C \mid G)=P(C \cdot G) / P(G)
$$

where $P_{G}$ and $P_{C}$ represent the probability of trap generation and trap charging respectively, $G$ and $C$ are trap generation and charging events respectively, and $P(C \bullet G)$ is the probability of $C$ and $G$ taking place at the same time. $P(C \mid G)$ in (5) is the probability of charging a trap at a location $(\mathrm{x}, \mathrm{y}, \mathrm{z})$ given a trap is being generated at the same location. Since $C$ is a subset of $G,(5)$ can be simplified to

$$
P(C \mid G)=P(C) / P(G)
$$

In order to model the conditional probability $P(C \mid G)$, we calculated the tunneling probability of electrons, $P_{W K B}$ by Wentzel-Kramers-Brillouin (WKB) method [24] and compared that with a set of pseudo-random probabilities $P_{r}$.

$$
P_{W K B}=\exp \left(-\frac{2}{\hbar}\left[\int_{0}^{z} \sqrt{2 m_{d e}^{*}\left(\phi_{B e}-E_{k}\right)} d z\right]\right)
$$

where, $m^{*}{ }_{d e}, \phi_{B e}, \hbar, E_{k}$ are the effective mass of electron in dielectric layers, effective barrier height seen by the tunneling electron, reduced Planck's constant, and the average kinetic energy of electron in a 3D semiconductor, respectively. $P_{r}$ is generated in such a way that they have the same order of magnitude as that of $P_{W K B}$. Once we have $P_{r}$ and $P_{W K B}, P(C \mid G)$ can be modeled as follows:

$$
P(C \mid G)=\left\{\begin{array}{l}
1: \text { if } P_{W K B}>P_{r} \\
0: \text { Otherwise }
\end{array}\right\}
$$

Since we can extract the locations of the generated traps from our simulation, $P(G)$ is either 0 or 1 if a bond is tied or broken, respectively. In addition, $P(C)=0$ if the bond is tied. Therefore, at a certain time $t,(6)$ becomes:

$$
P(C)_{t}=P(C \mid G)_{t}
$$

We calculate the total number of charged traps $\left(N_{c}\right)$ using the following expression:

$$
N_{C}(t)=\int_{x=1}^{W} \int_{y=1}^{L} \int_{z=1}^{T_{d i}} P(C)_{t, x, y, z} d x d y d z
$$

From the total number of charged traps $N_{C}(t), \Delta V_{t h}$ can be determined using poison's equation [11] or with the aid of the TCAD simulator [12].

The total $V_{\text {th }}$ degradation due to PBTI is dominated by the trap generation in the $\mathrm{HK}$ layer since the trap generation rate in the IL layer is very small and becomes significant only at a very high stress condition. We have assumed a Poisson distributed number of traps among a large number of samples in this work. For a more detailed analysis, the readers are referred to [11].

\section{Time-Dependent Dielectric Breakdown (TDDB)}

The time-dependent dielectric oxide breakdown (TDDB) in 


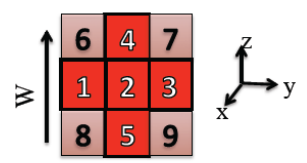

Top-view

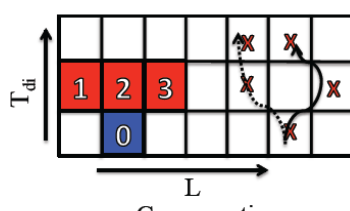

Cross-section

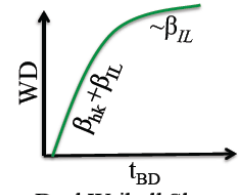

Dual Weibull Slope in HKMG transistor
Fig. 4: Implementation of the 3D percolation model. One single defect in the bottom layer can lead to multiple breakdown paths. ' $\mathrm{X}$ ' indicates a defect generated in a cell. Dual Weibull slope arises due to the different defect generation rates in the HK and IL layers.

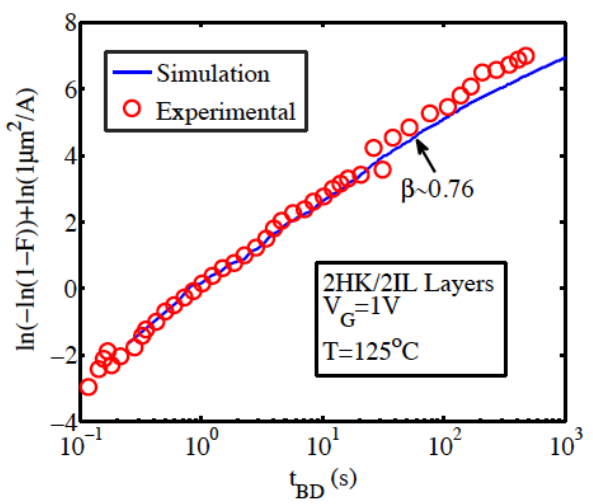

Fig. 5: The Weibull slope depends on the trap generation rate of the IL layer. For this simulation, the trap generation rate in the HK layer is assumed 50 times higher than the IL layer [17].

HKMG transistors is characterized by short breakdown times as well as shallow Weibull slopes [17]. We have incorporated a 3D percolation model into our BTI model in order to capture the TDDB effect in the IL and the HK layers. Although the high- $\kappa$ materials demonstrate lower tunneling current and improved transistor performance, a thin interfacial layer (IL) of $\mathrm{SiO}_{2}$ is needed to passivate the $\mathrm{Si}$-dielectric interface [17]. Since the defect generation rate in $\mathrm{SiO}_{2}$ at operating voltage is lesser than the high- $\kappa$ layer [13], the presence of this IL layer improves both TDDB and PBTI and also increases the carrier mobility $[13,17]$.

The formation of conduction paths due to the accumulation of oxide traps triggers the breakdown of a dielectric layer. The widely accepted percolation model [25] captures this idea for thick oxide layers. However, in scaled technologies, the formation of $3 \mathrm{D}$ conduction paths can no longer be ignored since the oxide layers are comprised of very few defect layers. Subsequently, 3D cell-based percolation model has been proposed and implemented in different literature [17,26]. In our statistical model, we have incorporated this model in order to capture the TDDB effect. The Weibull plot is determined as:

$$
W=\ln (-\ln (1-F))
$$

where, $F$ is the fraction of failed devices in a Monte Carlo (MC) event. Fig. 4 shows the cross section and top-view of a three-layer dielectric material and the dual Weibull slope observed in HK gate stacks. We have considered all of the nearest neighbors in determining a possible link between the two adjacent layers. In Fig. 4, a defect ' 0 ' in the bottom layer can have nine possible connections to the layer above itself. Defects ' 1 ', '2', ' 3 ', '4', and ' 5 ' have at least one common edge with ' 0 ', while defects ' 6 ', '7', ' 8 ', and '9' have exactly one node in common with ' 0 '. When a continuous path is established between the top and bottom layers, a conduction or breakdown path is considered to have formed. In the case of the dual-layer stack that is widely used in today's technology, TDDB analysis has become more complicated since high- $\kappa$ and interfacial layers have inhomogeneous defect generation rates. High- $\kappa$ layers are prone to the formation of both native and stress induced defects. Hence, they wear out relatively faster than the IL layer and the ultra-thin IL layer determines the final catastrophic breakdown [27]. The Weibull slope $\beta$ in the dielectric layers is defined as [17]:

$$
\beta=\alpha\left(\frac{T_{o x}}{a_{0}}\right)
$$

where, $\alpha$ is the time exponent of defect generation, $a_{0}$ is the cell or defect size, and $T_{o x}$ is the thickness of the either HK or IL layer. In order to validate the model, we did MC simulation with 10000 samples at $1 \mathrm{~V}$ and $125^{\circ} \mathrm{C}$ stress condition. We assumed a 50 times higher trap generation in the HK layer with a power exponent of 0.38 and the results are verified by the experimental results in [17]. The slope of the Weibull plot $(\sim 0.76)$ indicates that the final breakdowns depend on the two layers of defects in the IL layer. However, the actual reason behind the bi-modal distribution observed by different groups is still controversial. Yew, et al. [28] demonstrated that the presence of grain boundary (GB) defects in the HK layer can cause early breakdown in many devices and can give rise to similar bi-modal Weibull distribution. We have also considered this effect in our simulations in the results and discussion section.

\section{Stress-Induced Leakage Current (SILC)}

SILC is a means to analyze the buildup of oxide traps under BTI stress. As the trap density reaches a critical value, the SILC current can cause significant power consumption, leading to the hard breakdown of a device [29]. Therefore, it is necessary to understand the evolution of SILC and have appropriate model that can predict the progressive wear of the oxide layers in a scaled technology. Fig. 6 shows the band diagram of an NMOS under inversion mode and the components of gate leakage currents. There are three major components of gate leakage [30]:

1. Direct Tunneling Current $\left(J_{D T}\right)$ or the time-zero gate leakage

2. Trap-Assisted-Tunneling (TAT) Current in preexisting traps $(J(T P))$

3. Stress-Induced Leakage Current $\left(J_{S I L C}(T G)\right)$ through Trap-Assisted-Tunneling in generated traps

The time-zero leakage current is known as direct tunneling current $\left(J_{D T}\right)$ while the stress-induced leakage current $\left(J_{S I L C}\right)$ is known as Trap-Assisted-Tunneling (TAT) current. $J_{S I L C}$ increases when a device is under electrical stress. We implemented the modified direct tunneling current for a high- $\kappa$ gate stack proposed in [31]: 


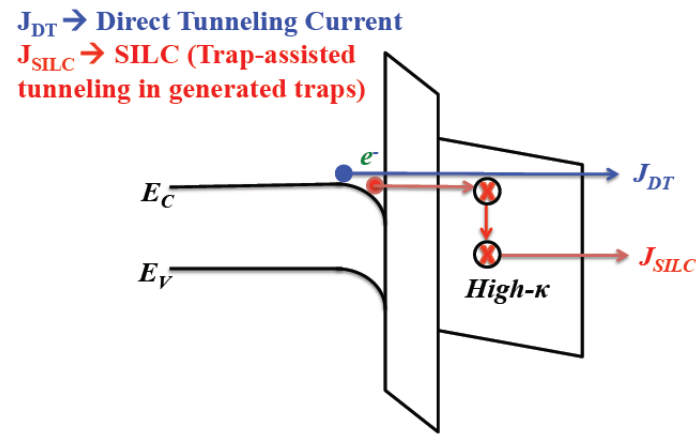

Fig. 6: Trap assisted SILC in HK layer decreases due to high relaxation energy.

$$
J_{D T}=\frac{q^{3}}{8 \pi h \phi_{B}} \frac{\phi_{B}}{V_{S i O_{2}}}\left(\frac{2 \phi_{B}}{V_{S i O_{2}}}-1\right) \frac{V_{S_{i O}}}{t_{S i O_{2}}} T_{W K B}
$$

where, $\Phi_{B}$ is the barrier height seen by the carriers in the conduction band of the substrate, $V_{\mathrm{SiO} 2}, t_{\mathrm{SiO} 2}$, and $T_{W K B}$ are the voltage drop across the $\mathrm{SiO}_{2}$ layer, thickness of the IL layer, and the tunneling probability across the dual gate stack determined using the WKB approximation [24], respectively. The stress-induced current due to trap generation (TG) is modeled as [13]

$$
\begin{array}{r}
J_{T G}(t)=\iint k \sigma_{n} V_{t h} N(t) T_{r}\left(f_{c}-f_{a}\right) d x d E \\
T_{r}=\frac{T_{\text {in }}(E) T_{\text {out }}\left(E-E_{r}\right)}{T_{\text {in }}(E)+T_{\text {out }}\left(E-E_{r}\right)}
\end{array}
$$

where, $T_{\text {in }}$ and $T_{\text {out }}$ are the tunneling probabilities to and from the traps, respectively; $E_{r}$ is the trap relaxation energy, $f_{c}$ and $f_{a}$

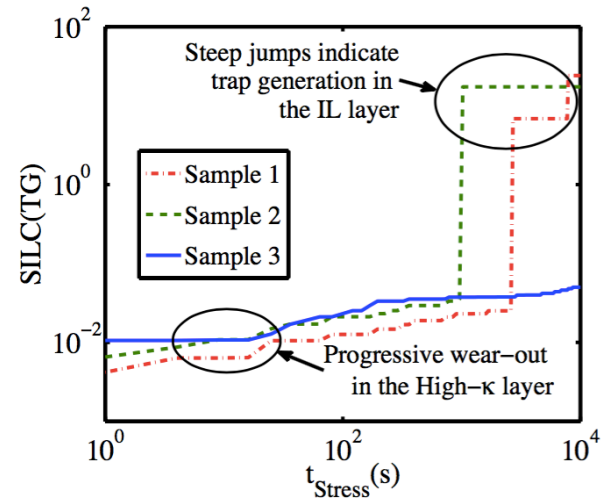

(a)

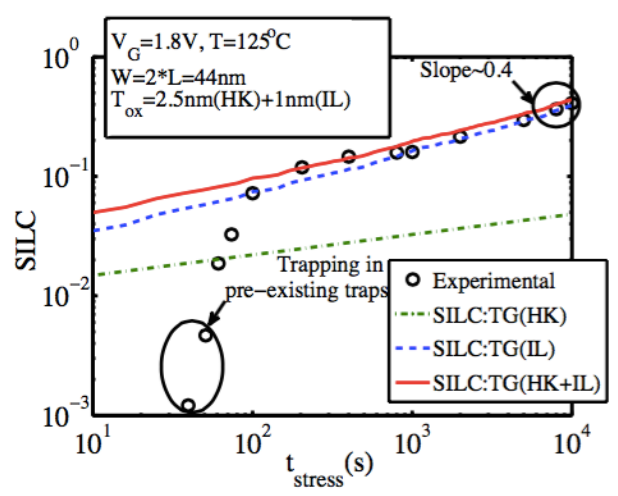

(b)

Fig. 7: (a) SILC in different small area sample devices indicates that trap generation in IL layer dominates total SILC; (b) Average SILC in 15000 such devices considering only trap generation is in close agreement the experimental data [13].

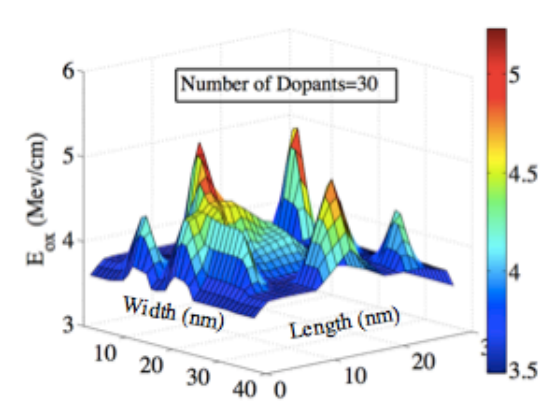

(a)

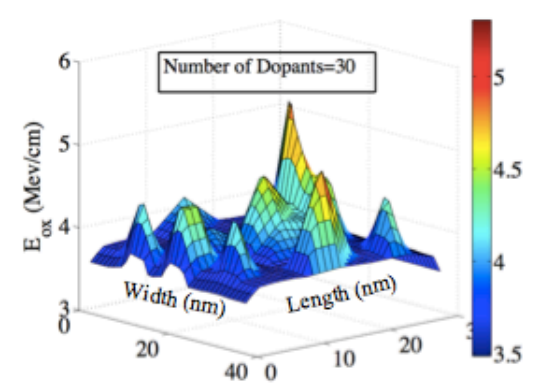

(d)

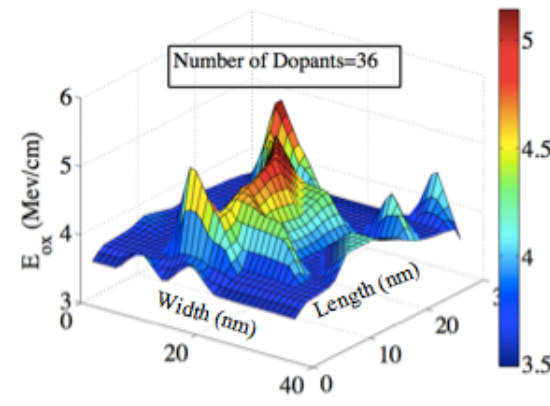

(b)

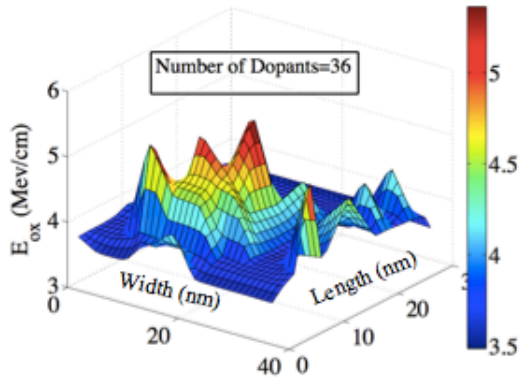

(e)

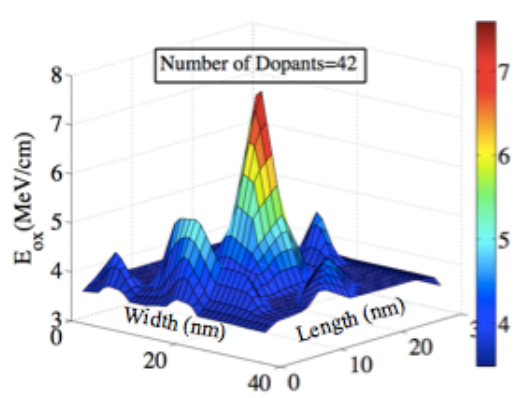

(c)

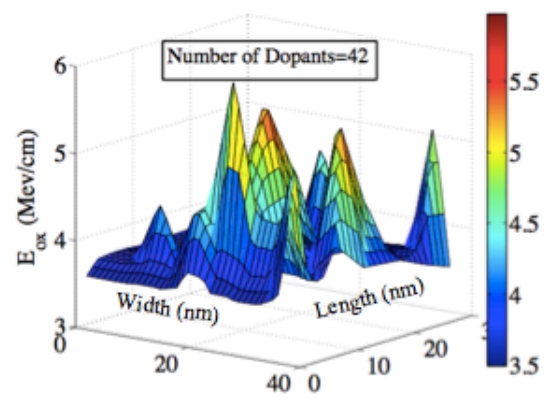

(f)

Fig. 8: Effect of random dopant fluctuation on the electric field across the IL oxide layer: (a), (b), and (c) show the electric field variation due to the fluctuation of the number of dopants in the channel region of an NMOS device. In comparison to the upper three figures, (d), (e), and (f) show the variation due to the fluctuation in dopant locations. A gate voltage of $1 \mathrm{~V}$ is applied in these simulations. 


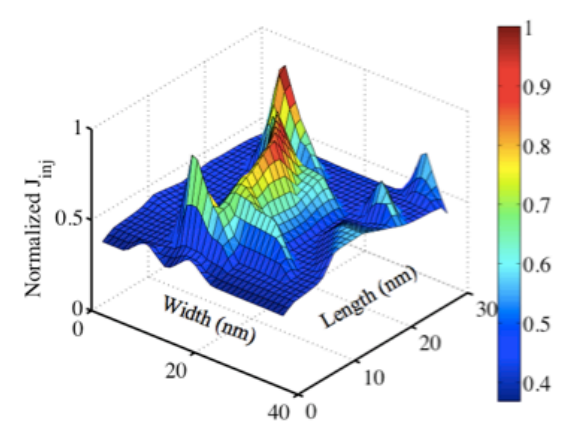

(a)

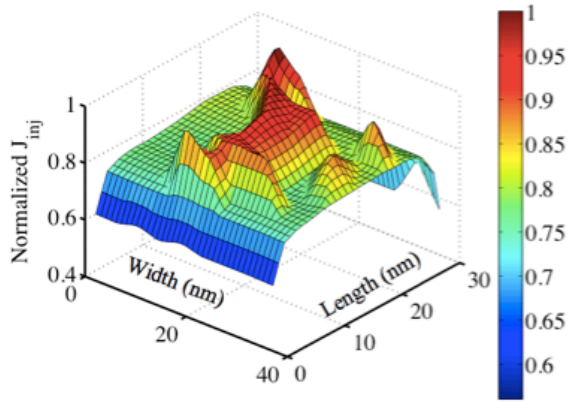

(b)

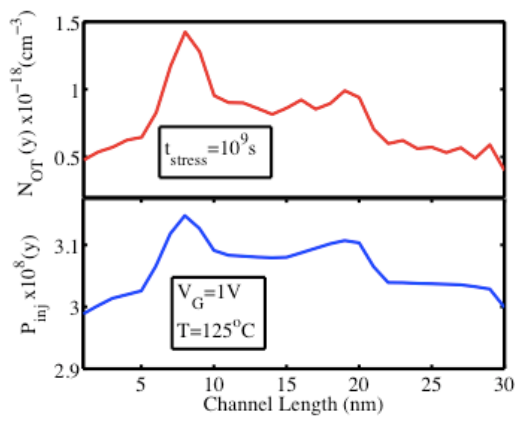

(c)

Fig. 9: Distribution of local injection current with 36 dopants inside the channel region: (a) $V_{G}=1 \mathrm{~V}$; (b) $V_{G}=2 \mathrm{~V}$; (c) Correlation between local injection probability $\left(P_{i n j}\right)$ and PBTI trap density $\left(\mathrm{N}_{\mathrm{OT}}\right)$ along the channel at $\mathrm{V}_{\mathrm{G}}=1 \mathrm{~V}$. The results indicate direct correlation between RDF and PBTI. The correlation is expected to be higher at a lower gate voltage.



Fig. 10: Effect of RDF on PBTI at a mean $\Delta V_{\text {th }}$ of $50 \mathrm{mV}$. Ignoring the effect of time-zero variability leads to erroneous results in BTI estimation.

are the occupation factors of cathode and anode, respectively; $\sigma_{n}$ is the electron capture cross section of the traps, and $v_{t h}$ is the thermal velocity. $k$ in (6) depends on the electron concentration of the conduction band and energy suppression factor. In our work, we have calibrated $k$ in order to fit the experimental data in [13]. The normalized SILC current is then defined as [32]

$$
J_{S L C}(t)=\frac{J_{T G}(t)-J_{T P}\left(t_{s a t}\right)}{J_{0}}
$$

Here $t_{\text {sat }}$ is the saturation time of pre-existing trap filling, which is usually in sub-milliseconds [33], and $J_{0}$ is the gate leakage current at $t=0$, which includes the direct-tunneling component and tarp-assisted tunneling in the pre-existing defects. The actual reason behind SILC is a controversial issue. While some groups attribute the SILC phenomenon to the defect generation in the HK layers [30, 34, 35], others explain SILC in terms of defect generation in the IL layer only [27, 36]. J. Yang, et al. [13] argued that SILC is caused by defects in both HK and IL layers but mostly dominated by IL layer defects with much lower relaxation energy. In our model we have considered degradation in both HK and IL layers and calibrated our model accordingly [13]. Fig. 7 shows the average SILC current for a MC simulation of 15000 similar devices. As we can observe from Fig. 7(a), for different small area sample devices, total SILC due to trap generation spikes when there is defect generation in the IL layer. This is because the relaxation energy in an IL defect is much lower than that of an HK defect [13]. The higher relaxation energy in the HK layer defects can exponentially decrease the tunneling out $\left(T_{\text {out }}\right)$ probability and significantly decrease the contribution to the overall SILC. As a result, the slope of the average SILC (Fig. 7(b)) gradually approaches the time exponent of trap generation in the IL layer. We have ignored the contribution from pre-existing traps and assumed $J_{T P}=0$ for our analysis since the trap density is not provided in [13]. Besides, these technology-dependent native traps can be optimized to a very low density [37], in which case only the trap generation plays a role in the SILC measurements.

\section{RESUlts AND Discussions}

In this section, we explain the impact of RDF on time dependent degradation using the results of our simulation framework. We extracted the electric field from the TCAD simulator. Fig. 8 shows the electric field variation across the IL layer among different devices with an average doping density of $2.7 \times 10^{18} / \mathrm{cm}^{3}$ in the channel region at $\mathrm{V}_{\mathrm{G}}=1 \mathrm{~V}$. We see variations in the oxide electric field due to the randomization of dopant locations as well as the numbers. As a result, we expect to see the impact of RDF in stress induced trap density profiles among a large number of microscopically similar devices. We incorporated the extracted electric field in our PBTI model [11] in order to further investigate the impact of RDF on different aging effects. In Fig. 9, we have plotted the local gate injection current as a function of channel length and width. The local injection current is determined using the direct tunneling current model in equation (13), and we plotted the normalized value (normalized with respect to the maximum value) for gate voltages of 1 and $2 \mathrm{~V}$ in Fig. 9(a) and 9(b), respectively. For this simulation, we have considered the dopant profile corresponding to Fig. 8(a), where 36 dopants are randomly assigned inside the channel. We can see that RDF causes variation in the local injected current at both $1 \mathrm{~V}$ 
and $2 \mathrm{~V}$. In order to investigate the relative impact of RDF, we determined the standard deviation of the local injected currents as a percentage of their average values. We observe that the variability induced by $\mathrm{RDF}$ at $1 \mathrm{~V}(21.9 \%)$ is much higher than compared to that at $2 \mathrm{~V}(10.3 \%)$. Therefore, the impact is expected to be more significant at the device operating voltage. Since PBTI is driven by the gate injection current, we investigated its correlation to local injected current probability $\left(P_{i n j}\right)$ in Fig. $9(\mathrm{c})$. For $\mathrm{V}_{\mathrm{G}}=1 \mathrm{~V}$ (corresponding to Fig. 9a), we extracted the probability as a function of the channel length at a random location along the width and incorporated the corresponding electric field distribution into our PBTI model. We simulated a large area device (Area $=10000 \times 30 \mathrm{~nm}^{2}$ ) for a stress period of $10^{9} \mathrm{~s}$ and plotted the lateral distribution of the generated trap density in the upper panel of Fig 9(c). In the lower panel, we have plotted the corresponding local injection probability and observe that they are closely correlated. Therefore, we conclude from Fig. 9 that the impact of RDF can modulate the $\Delta V_{T H}$ distribution induced by PBTI.

Fig. 10 demonstrates the impact of RDF on PBTI estimation for $50 \mathrm{mV}$ of mean $\Delta V_{t h}$ degradation. The lower panel represents the data where the BTI degradation is estimated using an average time-zero threshold voltage; the upper panel shows the same data taking the effects of RDF into account. $\Delta V_{\text {th }}$ after a stress period of $t_{\text {stress }}$ for these two panels are defined as:

$$
\Delta V \operatorname{th}\left(t_{\text {stress }}\right)=\left\{\begin{array}{l}
V t h\left(t_{\text {stress }}\right)-V t h(0), \text { upper pannel } \\
V t h\left(t_{\text {stress }}\right)-\mu_{V t h(0)}, \text { lower pannel }
\end{array}\right.
$$

Both sets of data are plotted against the difference between the time-zero threshold voltage, $V_{t h}(0)$ for each of the devices and the mean of the time zero threshold voltage, $\mu_{V t h}(0)$. In this plot, the devices on the left side are relatively faster, and we see that $\Delta V_{t h}$ for these devices are higher in the upper panel. The effect of RDF on the devices that have threshold voltages close to the mean $\mu_{V t h}(0)$ are almost negligible. The devices on the right side of the panels, on the other hand, have higher time-zero threshold voltages. We see that the $\Delta V_{t h}$ in the upper panel is relatively less than that of the lower panel. In other words, RDF causes faster degradation to the devices at the lower distribution tail as we have explained in Fig. 1.

Fig. 11(a) shows the impact of RDF on the threshold voltage degradation due to PBTI at three different stress conditions: $1.8 \mathrm{~V}$ and $105^{\circ} \mathrm{C} ; 1.8 \mathrm{~V}$ and $125^{\circ} \mathrm{C}$; and $2 \mathrm{~V}$ and $125^{\circ} \mathrm{C} . \sigma P B T I\left(V_{t h}(0)\right)$ is the PBTI standard deviation, taking the effect of RDF into account, while $\sigma P B T I\left(\mu V_{t h}(0)\right)$ represents PBTI variability considering average time-zero $V_{t h}$. We defined a dependence parameter $\zeta_{D}$, which is the underestimation of $\sigma_{\triangle V t h(P B T I)}$ with respect to mean $\Delta V_{t h(P B T I)}$ when the effect of RDF is ignored and plotted Fig. 11(b). We observe that the RDF effect on the threshold voltage degradation decreases with increased stress conditions. This trend follows the experimental data presented in [9]. This observation can be significant for scaled technology where the margin for parametric variation is very stringent [38]. In addition, we have plotted the standard deviation due to PBTI for different area of the devices in Fig. 12. The x-axis represents (Area) $)^{-0.5}$, and the variation shows a linear relationship. This confirms that the PBTI degradation follows Pelgrom's rule [42] when the effect of RDF is taken into consideration. However, the impact of correlation becomes weaker with the increase of deice area.

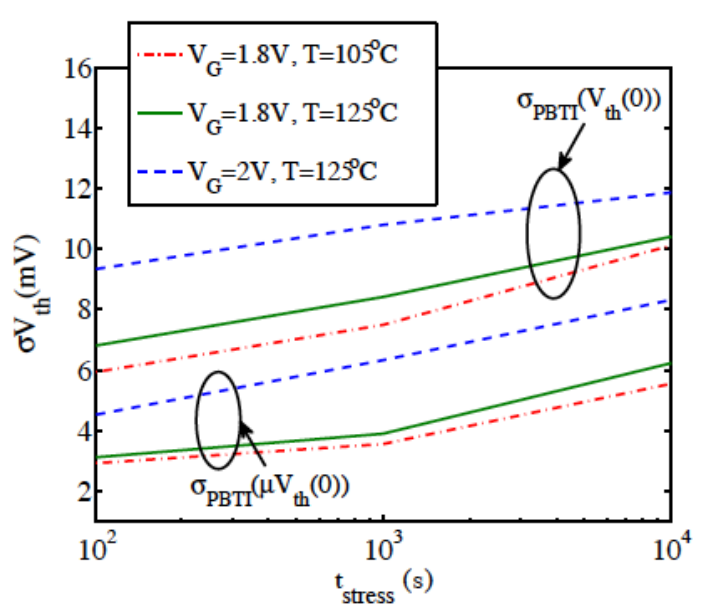

(a)

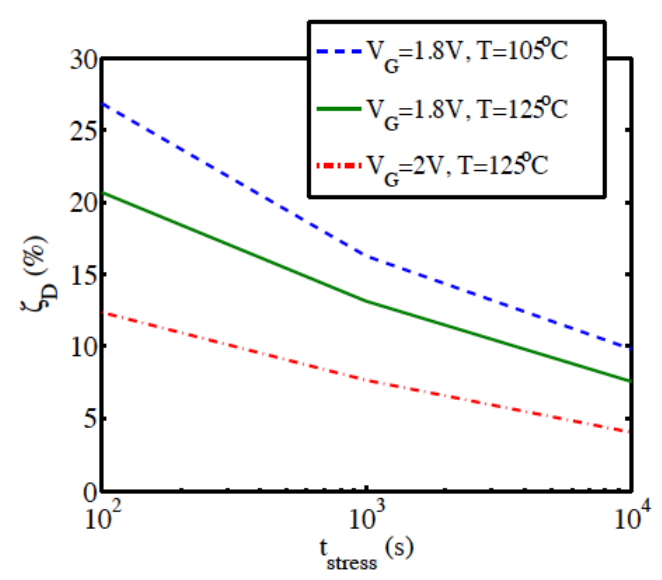

(b)

Fig. 11: (a) PBTI degradation for various stress conditions; (b) Effect of RDF on PBTI decreases with increased BTI degradation.

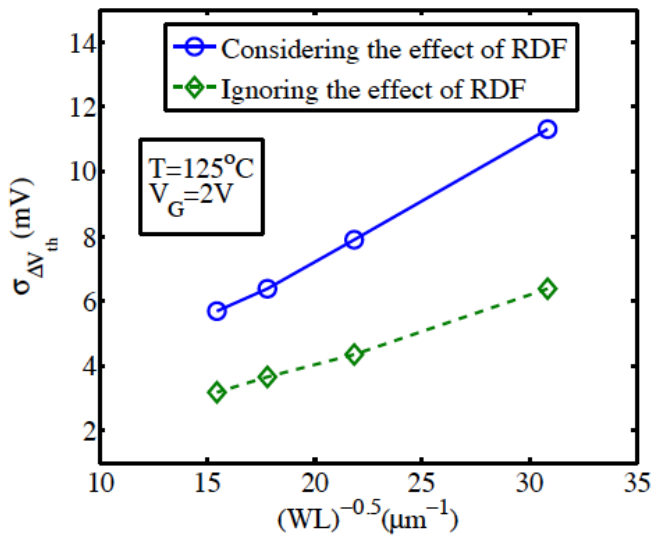

Fig. 12: The variation due to PBTI follows Pelgrom's rule [42] regardless of the PBTI-RDF correlation. 


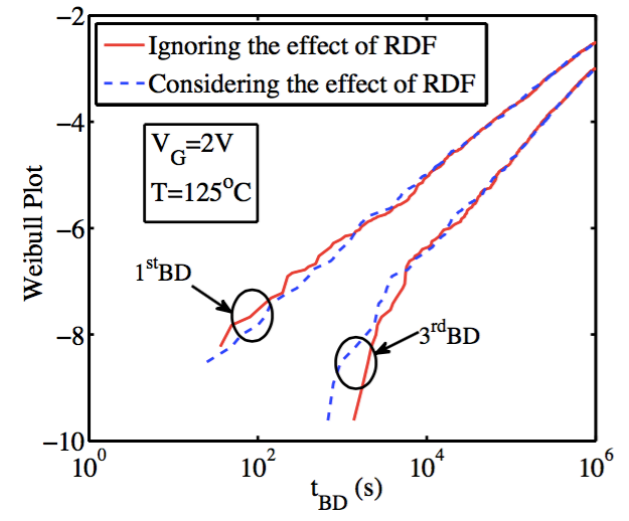

Fig. 13: The effect of RDF has very negligible or no impact on TDDB estimation.

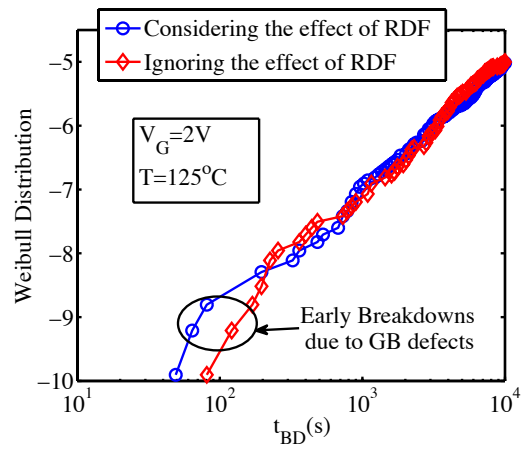

Fig. 14: Impact of RDF on TDDB, taking GB defects into account. The effect of RDF on TDDB is still negligible.

Next, we investigated the effect of RDF on TDDB and SILC at a stress voltage of $2 \mathrm{~V}$. The analysis was carried out for 15000 sample devices. Fig. 13 shows the Weibit plot for both the $1^{\text {st }}$ and the $3^{\text {rd }}$ breakdown with and without considering the effect of RDF. We observe negligible differences in the TDDB lifetime. We carried out similar analysis, taking the presence of GB defects into consideration. We divided the HK oxide layer in 42 grain-regions and assigned an average pre-existing GB defect density of $10^{19} / \mathrm{cm}^{3}$ in $25 \%$ of the sample devices. Since the presence of GB defects compromises the underlying IL layer [28], we assumed a 10 times higher IL layer defect generation rate in these devices. The simulation results for the $3^{\text {rd }}$ soft breakdown is plotted in Fig. 14, and we observe the negligible effect of RDF on TDDB. Early breakdowns due to GB defects in some of the devices give rise to a bi-modal Weibull distribution, which is consistent with the observations reported in [28]. Since TDDB requires higher stress conditions and defect density than BTI, the effect of RDF is negligible in both Fig. 13 and 14.

A similar trend is observed in SILC analysis and shown in Fig. 15. We observe almost indistinguishable SILC for both average doping effect and RDF. SILC is dominated by the trap generation in the IL layer, where the defect generation rate is assumed to be very small in our simulations. Due to the low density of generated traps in the IL layer, the statistical fluctuation due to the RDF effect has a trivial effect on SILC.

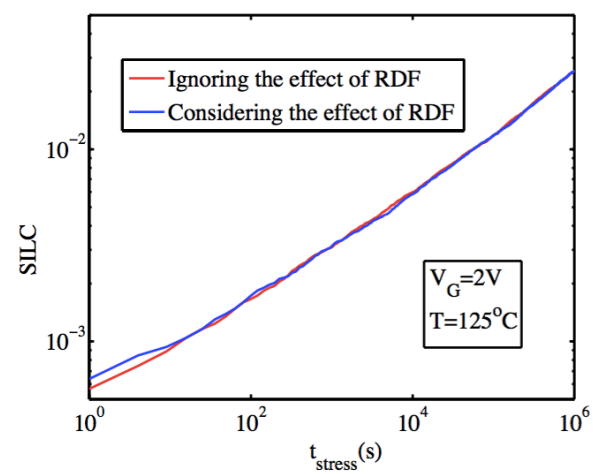

Fig. 15: Average SILC shows similar trend in TDDB since both are dominated by the trap generation rate of the IL layer.

Table 3: NBTI model used in our work

The average number of interface traps generated is predicted by the following $\mathrm{H}-\mathrm{H}_{2} \mathrm{R}-\mathrm{D}$ model [43]

$$
\begin{gathered}
N_{I T_{\text {avg }}}(t)=W L\left(k_{H} / k_{H_{2}}\right)^{1 / 3}\left(k_{f}(y) N_{0} / k_{r}\right)^{2 / 3}\left(6 D H_{2} t\right)^{n} \\
k_{f}(y)=k_{f 0} E c \exp (\gamma \operatorname{Eox}(y)) \exp \left(-\frac{E A_{k_{f}}}{q V_{T}}\right)
\end{gathered}
$$

Where, $k_{f}, k_{r}, N_{0}, k_{H}, k_{H 2}$, and $D_{H 2}$ are $\mathrm{Si}-\mathrm{H}$ bond breaking rate, $\mathrm{Si}-\mathrm{H}$ bond annealing rate, the pre-stress $\mathrm{Si}-\mathrm{H}$ bond density at the $\mathrm{Si} / \mathrm{SiO}_{2}$ interface, generation rate, dissociation rate, and diffusion coefficient for $\mathrm{H}$ and $\mathrm{H}_{2}$. y in the above equation is the distance along the channel length. $k_{f 0}$ is the calibration parameter.

In the above equation,

$$
\begin{aligned}
& X=X_{0} e^{-\frac{E A_{X}}{q V_{T}}} \\
& X=\left\{k_{f}, k_{r}, k_{H}, k_{H_{2}}, D_{H_{2}}\right\}
\end{aligned}
$$

$E A_{x}$ are the activation energies and $X_{0}\left\{=k_{f 0}, k_{r 0}, k_{H 0}, k_{H 20}\right.$, $\mathrm{DH}_{20}$ \} are constants

$\mathrm{n}=1 / 6, \gamma=0.6 \pm 0.05, \mathrm{EF}=0.36 \mathrm{eV}[42]$

$\mathrm{EA}_{\mathrm{kf}}=0.175 \mathrm{eV}, \quad \mathrm{E}_{\mathrm{Akr}}=0.2 \mathrm{eV}, \quad \mathrm{E}_{\mathrm{AkH}}=\mathrm{EA}_{\mathrm{kH} 2}=0.3 \mathrm{eV}$, $\mathrm{EA}_{\mathrm{DH} 2}=0.58 \mathrm{eV}, \quad \mathrm{K}_{\mathrm{r} 0}=9.9 \times 10^{-7}, \quad \mathrm{D}_{\mathrm{H} 0}=9.56 \times 10^{-11} \mathrm{~cm}^{2} / \mathrm{s}$, $\mathrm{K}_{\mathrm{H} 0}=8.56 \mathrm{~cm}^{3} / \mathrm{s}, \mathrm{K}_{\mathrm{H} 20}=5.7 \times 10^{5} / \mathrm{s}, \mathrm{D}_{\mathrm{H} 20}=3.5 \times 10^{-5} \mathrm{~cm}^{2} / \mathrm{s}[46]$

SILC is highly a controversial issue, and the relative contribution of stress-induced traps in the HK and IL layers is still debatable. Therefore, further investigation on the effect of RDF on SILC is necessary.

Finally, we investigated the effect of RDF on BTI degradation at the circuit level and analyzed the impact of the RDF-BTI correlation on the degradation in the frequency of oscillation $\left(f_{o}\right)$ and transient supply current $\left(I_{D D T}\right)$ of ring oscillators. Both parameters are expected to decrease over time since transistor's speed and sub-threshold leakage current decrease due to BTI effects [45]. We extracted I-V characteristics from the TCAD simulator and implemented a look-up table based Verilog-A model for both $\mathrm{n}$ and $\mathrm{p}$ MOSFETs. Using the models, we carried out HSPICE Monte Carlo simulations on 1000 samples. For NBTI degradation in PMOS transistors, we implemented the reaction-diffusion 
(RD) model [43] and assumed Poisson's distribution on the interface trap numbers among different microscopic samples [44]. The average number of interface traps was calibrated by $\mathrm{K}$. Kang et al. [45]. The RD model used in our work is summarized in Table 3. We have used the same framework, as shown in Fig. 2, to take the effect of RDF on NBTI into account. In order to explore the performance degradation of the relatively slower circuits, we determined the $-3 \sigma$ points of the above-mentioned parameters with and without considering the effect of RDF on BTI and plotted their difference as a percentage of the standard deviation $(\sigma)$ in Fig. 16. We observe that ignoring the effect of RDF causes significant underestimation of both $f_{o}$ and $I_{D D T}$, especially at a relatively lower BTI degradation. Similar to the device level results, the influence of RDF decreases as BTI degradation increases. Therefore, the effect of RDF on BTI may have to be considered in scaled technology where the variability margin is strict.

\section{CONCLUSION}

In this paper, we have proposed a statistical modeling framework that takes the dependence between time-zero variability and various time-dependent aging effects into account. The framework is developed based on our proposed work of PBTI, a statistical RD model of NBTI, a 3D cell based percolation model of TDDB, and a TAT current model of SILC. We have analyzed small area devices $\left(\sim 1000 \mathrm{~nm}^{2}\right)$ and shown that: 1) RDF has considerable impact on BTI effect; and 2) the influence of RDF on time dependent oxide breakdown is almost negligible. In addition, we carried out circuit analysis using a look-up table based Verilog-A SPICE model and analyzed the effect of BTI degradation on the performance of ring oscillators. On both the device and circuit levels, we observed that the effect of RDF on BTI degradation is more pronounced at relatively lower BTI degradation. Since there are not enough experimental evidence that proves any impact of RDF on TDDB and SILC, further investigation is necessary. We conclude that the effect of RDF on $V_{t h}$ degradation needs to be properly addressed in scaled CMOS technology, and this may become crucial in the accurate guard band estimation of a circuit.

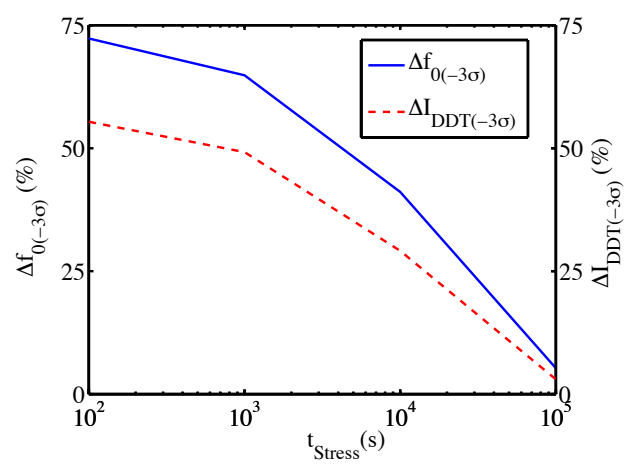

Fig. 16: $-3 \sigma$ points of frequency of oscillation and dynamic supply current as a percentage of their corresponding standard deviations. Effect of RDF on BTI effect is more noticeable at lower BTI degradation

\section{ACKNOWLEDGEMENT}

This work is funded in part by the National Science Foundation.

\section{REFERENCES}

[1] J. Hicks, D. Bergstrom, M. Hattendorf, J. Jopling, J. Maiz, S. Pae, C. Prasad, and J. Wiedemer, " $45 \mathrm{~nm}$ Transistor Reliability," Intel Technol. J., vol. 12, no. 2, pp. 131-144, 2008.

[2] K. Mistry and et al, "A 45nm Logic Technology with High$\kappa+$ Metal Gate Transistors, Strained Silicon, $9 \mathrm{Cu}$ Interconnect Layers, $193 \mathrm{~nm}$ Dry Patterning, and $100 \% \mathrm{~Pb}-$ free Packaging," in Proc. IEDM Tech. Dig., 2007, pp. 247250.

[3] X. Chen and et al, "A cost effective 32nm high- $\kappa /$ metal gate CMOS technology for low power applications with single-metal/gate-first process," in Proc. Symp. VLSI Technol., 2008, pp. 88-89.

[4] A. Kerber and E. Cartier, "Reliability Challenges for CMOS Technology Qualifications With Hafnium Oxide/Titanium Nitride Gate Stacks," IEEE Trans. Device Mater. Rel., vol. 9, no. 2, pp. 147-162, 2009.

[5] International Technology Roadmap for Semiconductors, 2009.

[6] R. Degraeve, M. Aoulaiche, B. Kaczer, P. Roussel, T. Kauerauf, S. Sahhaf, and G. Groeseneken, "Review of reliability issues in high-к /metal gate stacks," in IEEE Int. Symp. Physical and Failure Analysis of Integrated Circuits, 2008, pp. 1-6.

[7] J. Robertson, "High dielectric constant gate oxides for metal oxide Si transistors," Rep. Progr. Phys., vol. 69, no. 2, pp. 327-396, 2006.

[8] G. Bersuker, J. Sim, C. S. Park, C. D. Young, S. Nadkarni, R. Choi, and B. H. Lee, "Mechanism of Electron Trapping and Characteristics of Traps in $\mathrm{HfO}_{2}$ Gate Stacks," IEEE Trans. Device Mater. Rel., vol. 7, no. 1, pp. 138-145, 2007.

[9] A. Kerber and T. Nigam, "Challenges in the characterization and modeling of BTI induced variability in metal gate / High-к CMOS technologies," in Proc. IEEE IRPS, 2013, pp. 2D.4.1-2D.4.6.

[10] T. Grasser, Bias Temperature Instability for Devices and Circuits, $1^{\text {st }}$ ed. Springer Publishing Company, Incorporated, 2014.

[11] M. K. Hassan, C. Ho, and K. Roy, "Stochastic Modeling of Positive Bias Temperature Instability in High- $\kappa$ Metal Gate nMOSFETs," IEEE Trans. Electron Devices, vol. 61, no. 7, pp. 2243-2249, 2014.

[12] Sentaurus Device Simulator, G-2012.06 ed. Mountain View, California: Synopsys, Inc., 2012.

[13] J. Yang, M. Masuduzzaman, K. Joshi, S. Mukhopadhyay, J. Kang, S. Mahapatra, and M. Alam, "Intrinsic correlation between PBTI and TDDB degradations in nMOS HK/MG dielectrics," in Proc. IEEE IRPS, 2012, pp. 5D.4.1-5D.4.7.

[14] MATLAB and Statistics Toolbox Release 2013b, The MathWorks, Inc., Natick, Massachusetts.

[15] D. B. M. Klaassen, "A unified mobility model for device simulation-I. Model equations and concentration dependence," Solid-State Electron., vol. 35, pp. 953-959, 1992.

[16] M. J. V. Dort, P. H. Woerlee, A. J. Walker, "A simple model for quantisation effects in heavily-doped silicon MOSFETs 
at inversion conditions, "Solid-State Electron., vol. 37, pp. 411-414, 1994.

[17] T. Nigam, A. Kerber, and P. Peumans, "Accurate model for time-dependent dielectric breakdown of high- $\kappa$ metal gate stacks," in Proc. IEEE IRPS, 2009, pp. 523-530.

[18] L. Yiming, S. M. Yu, J. R. Hwang, and F. L. Yang, "Discrete Dopant Fluctuations in 20-nm/15-nm-Gate Planar CMOS," IEEE Trans. Electron Devices, vol. 55, no. 6, pp. 1449-1455, 2008.

[19] M. Toledano-Luque and et al, "Degradation of time dependent variability due to interface state generation," in Proc. Symp. VLSI Technol., 2013, pp. T190-T191.

[20] A. Asenov, A. Brown, J. Davies, S. Kaya, and G. Slavcheva, "Simulation of intrinsic parameter fluctuations in decananometer and nanometer-scale MOSFETs," IEEE Trans. Electron Devices, vol. 50, no. 9, pp. 1837-1852, 2003.

[21] A. Asenov, "Random Dopant Induced Threshold Voltage Lowering and Fluctuations in Sub-0.1 m MOSFET's: A 3-D "Atomistic" Simulation Study," IEEE Trans. Electron Devices, vol. 45, no. 12, pp. 2505-2513, 1998.

[22] G. Panagopoulos and K. Roy, "A physics-based 3-D analytical model for RDF induced threshold voltage variations," IEEE Trans. Electron Devices, vol. 58, no. 2, pp. 392-403, 2011.

[23] A. Papoulis and S. U. Pillai, Probability, Random Variables and Stochastic Processes, 4th ed. McGraw-Hill Publishing Company, Incorporated, 2001.

[24] H. Y. Yang, H. Niimi, and G. Lucovsky, "Tunneling currents through ultrathin oxide/nitride dual layer gate dielectrics for advanced microelectronic devices," J. Appl. Phys., vol. 83, no. 4, pp. 2327-2337, 1998.

[25] J. Stithies, "Percolation models for gate oxide breakdown," J. Appl. Phys., vol. 86, no. 10, pp. 5757-5766, 1995.

[26] J. Sune, S. Tous, and E. Wu, "Analytical Cell-Based Model for the Breakdown Statistics of Multilayer Insulator Stacks," IEEE Electron Device Lett., vol. 30, no. 12, pp. 1359-1361, 2009.

[27] G. Bersuker and et al, "Breakdown in the metal/high- $\kappa$ gate stack: Identifying the weak link in the multilayer dielectric," in Proc. IEDM Tech. Dig., 2008, pp.1-4.

[28] K. S. Yew, D. S. Ang, and G. Bersuker, "Bimodal Weibull Distribution of Metal/High- $\kappa$ Gate Stack TDDB_-Insights by ScanningTunneling Microscopy," IEEE Electron Device Lett., vol. 33, no. 2, pp. 146-148, 2012.

[29] L. Pantisano and K. Cheung, "Stress-induced leakage current (SILC) and oxide breakdown: are they from the same oxide traps?," IEEE Trans. Device Mater. Rel., vol. 1, no. 2, pp. 109-112, 2001.

[30] E. Cartier and A. Kerber, "Stress-induced leakage current and defect generation in nFETs with $\mathrm{HfO} 2 / \mathrm{TiN}$ gate stacks during positive bias temperature stress," in Proc. IEEE IRPS, 2009, pp. 486-492.

[31] G. Darbandy and et al, "Analytical modeling of direct tunneling current through gate stacks for the determination of suitable high- $\kappa$ dielectrics for nanoscale double-gate MOSFETs," Semicond. Sci. Technol., vol. 26, no. 4, pp. 1-8, 2011.

[32] D. DiMaria and E. Cartier, "Mechanism for stress-induced leakage currents in thin silicon dioxide films," J. Appl. Phys., vol. 78, no. 6, pp. 3883-3894, 1995.

[33] S. Mahapatra, V. Maheta, A. Islam, and M. Alam, "Isolation of NBTI Stress Generated Interface Trap and Hole-Trapping Components in PNO p-MOSFETs," IEEE Trans. Electron Devices, vol. 56, no. 2, pp. 236-242, 2009.

[34] F. Crupi, R. Degraeve, A. Kerber, D. H. Kwak, G. Groeseneken, "Correlation between Stress-Induced Leakage
Current (SILC) and the $\mathrm{HfO}_{2}$ bulk trap density in a $\mathrm{SiO} / \mathrm{HfO} 2$ stack", in Proc. IEEE IRPS, 2004, pp 181-187.

[35] S. Pae, T. Ghani, M. Hattendorf, J. Hicks, J. Jopling, J. Maiz, K. Mistry, J. O'Donnell, C. Prasad, J. Wiedemer, J. $\mathrm{Xu}$, "Characterization of SILC and its end-of-life reliability assessment on 45NM high- $\kappa$ and metal-gate technology", in Proc. IEEE IRPS, 2009, pp. 499-504.

[36] C. D. Young, G. Bersuker, M. Jo,et al., "New insights into SILC-based life time extraction," in Proc. IEEE Int. Rel. Phys. Symp., 2012, pp. 5D.3.1-5D.3.5.

[37] X. Garros and et al, "PBTI mechanisms in La containing Hfbased oxides assessed by very Fast IV measurements," in Proc. IEDM Tech. Dig., 2010, pp. 4.6.1-4.6.4.

[38] M. Alam, K. Roy, and C. Augustine, "Reliability-and Process-variation aware design of integrated circuits A broader perspective," in Proc. IEEE IRPS, 2011, pp. 4A.1.14A.1.11.

[39] R. Degraeve, B. Govoreanu, B. Kaczer, J. Van Houdt, andG. Groeseneken, "Measurement and statistical analysis of singletrap current-voltage characteristics in ultrathin SiON," inProc. IRPS, 2005, pp. 360-365.

[40] D. M. Ramo, J. L. Gavartin, A. L. Shluger, and G. Bersuker, "Spectroscopic Properties of Oxygen Vacancies in Monoclinic HfO2 Calculated with Periodic and Embedded Cluster Density Functional Theory," Phys. Rev. B (Condensed Matter and Materials Physics), vol.75, no. 20, p. 205336, 2007.

[41] R. Degraeve et al., "Trap Spectroscopy by Charge Injection and Sensing (TSCIS): A Quantitative Electrical Technique for Studying Defects in Dielectric Stacks," in IEDM Tech. Dig., 2008, pp. 1-4.

[42] M. J. M. Pelgrom, A. C. J. Duinmaijer, and A. P. G. Welbers, "Matching properties of MOS transistors," IEEE Journal of Solid-State Circuits, vol. 24, no. 5, pp. $1433\{1439$, Oct 1989.

[43] A. E. Islam, H. Kufluoglu, D. Varghese, S. Mahapatra, and M. A. Alam, "Recent issues in negative-bias temperature instability: Initial degradation, field dependence of interface trap generation, hole trapping effects, and relaxation," IEEE Trans. Electron Devices, vol. 54, no. 9, pp. 2143- 2154, Sep. 2007.

[44] S. E. Rauch, "The statistics of NBTI-induced VT and $\beta$ mismatch shifts in pMOSFETs," IEEE Trans. Device Mater. Rel., vol.2, no.4, pp.89-93, 2002.

[45] K. Kang, S. P. Park, K. Roy, and M. A. Alam, "Estimation of statistical variation in temporal NBTI degradation and its impact on lifetime circuit performance," in Proc. IEEE/ACM Int. Conf. Comput.-Aided Design, 2007, pp. 730-734.

[46] K. Joshi, S. Mukhopadhyay, N. Goel, and S. Mahapatra, "A Consistent Physical Framework for N and P BTIin HKMG MOSFET", in Proc. IEEE IRPS, 2012, pp. 5A.3.1-5A.3.10. 